\title{
Enhanced High Speed SE imaging in a VPSEM using a Frisch Grid
}

\author{
M. R. Phillips* and S.W. Morgan* \\ * Microstructural Analysis Unit, University of Technology, Sydney, PO Box 123, Broadway NSW \\ 2007 Australia
}

Secondary electron (SE) imaging in a variable pressure SEM has necessitated the development of new detector technologies. One approach is to measure the charge, $\mathrm{Q}$, induced on a positive electrode placed at some distance, $r$, from the specimen stage which is a ground potential. Gas ionization by sufficiently energetic SEs produces electron-ion pairs which are charge separated by the applied electric field. Electrons drift towards the anode causing further gas ionization in a cascade process, and the ions drift towards the stage (cathode). The total Q induced at the anode will include charge components induced by the motion of both electrons, QE, and ions, QI. From Ramo's theorem [1], the charge induced on an electrode by a point charge moving in an electric field will be proportional to the ratio of the distance traveled by the charge to $\mathrm{r}$. The motion of the electron and the ion will induce at total charge of $\Delta \mathrm{Q}_{\mathrm{T}}=\mathrm{Q}_{\mathrm{E}}+\mathrm{Q}_{\mathrm{I}}=\mathrm{qx}_{\mathrm{E}} \mathrm{r}^{-1}+\mathrm{q}\left(1-\mathrm{x}_{\mathrm{E}}\right) \mathrm{r}^{-1}=\mathrm{qx}_{\mathrm{E}} \mathrm{r}^{-1}+\mathrm{qx}_{\mathrm{I}} \mathrm{r}^{-1}$, where $\mathrm{q}$ is the electronic charge, $\mathrm{x}_{\mathrm{E}}$ is the distance the electron is displaced and $\mathrm{x}_{\mathrm{I}}$ is distance the ion is displaced. The total induced current, $\mathrm{I}_{\mathrm{T}}$, can be obtained from $\mathrm{I}_{\mathrm{T}}=\mathrm{dQ} \mathrm{Q}_{\mathrm{T}} / \mathrm{dt}=\mathrm{q}\left(\mathrm{x}_{\mathrm{E}} / \mathrm{dt}\right) \mathrm{r}^{-1}+\mathrm{q}\left(\mathrm{x}_{\mathrm{I}} / \mathrm{dt}\right) \mathrm{r}^{-1}=$ $\mathrm{qv}_{\mathrm{E}} \mathrm{r}^{-1}+\mathrm{qv}_{\mathrm{I}} \mathrm{r}^{-1}$, where $\mathrm{v}_{\mathrm{E}}$ and $\mathrm{v}_{\mathrm{I}}$ are the electron and ion drift velocity respectively. Since the $\mathrm{v}_{\mathrm{E}}$ is around three orders of magnitude greater that $\mathrm{v}_{\mathrm{I}}$ the total induced current pulse for each electron-ion pair consists of a sharp electron peak followed by a (much weaker) slow ion tail. The maximum width of this current pulse is determined by the time taken for the ions to traverse $\mathrm{x}_{\mathrm{I}}$ which is typically $1-100 \mu \mathrm{s}$, depending on $\mathrm{r}$ and $\mathrm{v}_{\mathrm{I}}$. Consequently, at fast scan speeds the current induced by ions is not completely collected during the pixel dwell time.

This problem has been addressed in detectors employed in nuclear physics by the use of a Frisch grid which is placed a distance, $z$, in front of the anode [2]. The grounded grid in this application serves two purposes. First, it electrostatically screens the anode from the movement of ions and electrons between the grid and the cathode, and second, it enables the electrons that cross the gap between the grid and the anode to induce their total charge without the involvement of the ions. By placing a Frisch grid below the anode in a VPSEM (ideally at a distance close to the gas ionization path length), the total induced current can in principle be collected at high scan rates without ion related artifacts, such as SE emission quenching and gas gain damping. SE gas gain below the grid can be achieved by biasing the specimen stage with a negative voltage to accelerate SEs towards the grid and ions towards the stage. The grounded grid also provides a pathway for ion recombination and efficiently removes any additional ions produced between the grid and the anode [3].

A grounded Frisch grid with a $1 \mathrm{~mm}$ spacing was placed between the anode and the sample stage in a FEI Quanta 200. A TV rate SE image of a uncoated sapphire specimen (pixel dwell time $=100 \mathrm{~ns}$ ) using the induced current collected at the anode biased at $+550 \mathrm{~V}$ with the grid grounded and the stage at $0 \mathrm{~V}$ is shown in Figure 1. A corresponding image collected under identical conditions with the grid grounded and the stage bias at $-120 \mathrm{~V}$ (Figure 2) was found to exhibit significant improvement, being virtually free of ion-related artifacts. Since the stage is negatively biased with respect to the grid, the reduction in ion related artifacts cannot be attributed solely to the reduction of ions in the chamber. Instead it is proposed that the enhancement in these SE images is due to a 
significant reduction in the ion contribution to the induced current collected at the anode. Highspeed SE imaging without ion-related artifacts is possible because ion motion below the grid is shielded from the anode and ions produced between the grid and the anode recombine on the grid with a reduced transit time. Equivalent images (not shown) collected with the grid removed exhibited an increase in edge smearing with increasing negative stage bias in contrast to the decrease in smearing observed with the grounded grid in place. Biasing the grid may enable optimization of the gas gain [4] in conjunction with a reduction in ion-related image artifacts.

References:

[1] S. Ramo, Proceedings of the Institute of Radio Engineers, 27 (1939) 584

[2] O. Frisch, British Atomic Energy Report, BR-49 (1944)

[3] J. P. Craven, F.S. Baker, B.L. Thiel and A.M. Donald, Journal of Microscopy, 205 (2002) 96

[4] G. Danilatos, Advances in Electronics and Electron Physics 78 (1990) 1

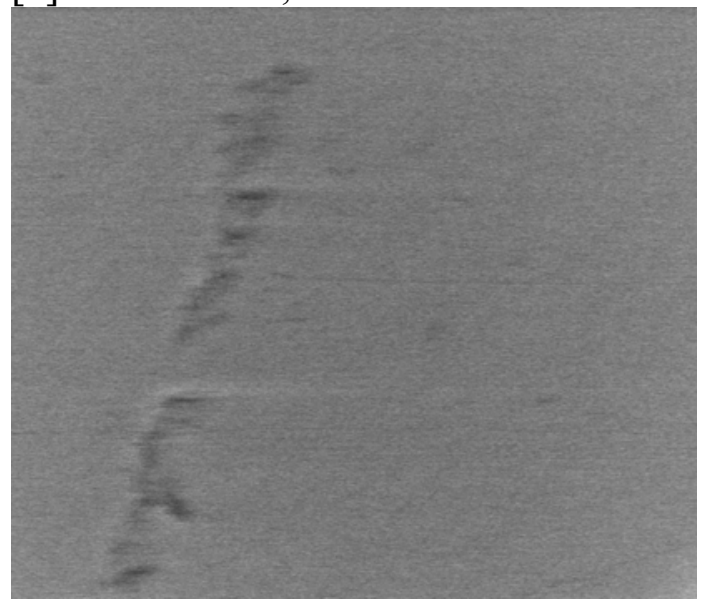

Fig. 1. TV rate SE image of an uncoated sapphire specimen using the induced anode current with the grid grounded and the stage at $0 \mathrm{~V}$, anode bias $=550 \mathrm{~V}$, pixel dwell time $=100 \mathrm{~ns}$, working distance $=13 \mathrm{~mm}$, grid position $=5 \mathrm{~mm}$ below the anode, Accelerating voltage $=30 \mathrm{kV}$, water vapor pressure $=1$ Torr. Horizontal width of field $=150 \mu \mathrm{m}$

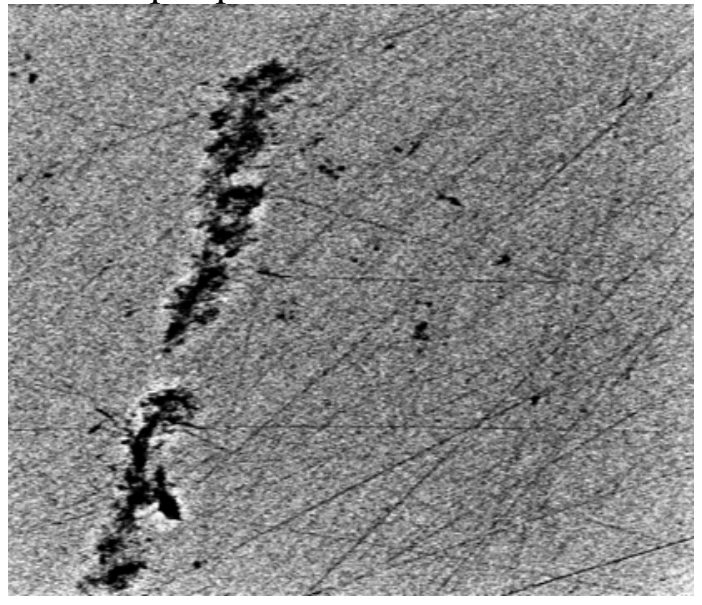

Fig. 2. TV rate SE image of an uncoated sapphire specimen using the induced anode current with the grid grounded and the stage at $-120 \mathrm{~V}$ collected under identical conditions to Fig 1. 